\title{
A CHARACTERIZATION OF THE LORENTZ SPACE $L(p, r)$ IN TERMS OF ORLICZ TYPE CLASSES
}

\author{
CALIXTO P. CALDERÓN AND ALBERTO TORCHINSKY
}

In remembrance of $N$. M. Rivière (1940-1978), who believed in Lorentz spaces.

\begin{abstract}
We describe the Lorentz space $L(p, r), 0<r<p, p>1$, in terms of Orlicz type classes of functions $L_{\Psi}$. As a consequence of this result it follows that Stein's characterization of the real functions on $\mathbb{R}^{n}$ that are differentiable at almost all the points in $\mathbb{R}^{n}$ [Ann. of Math 113 (1981), no. 2, 383-385], is equivalent to the characterization of those functions given by A. P. Calderón [Riv. Mat. Univ. Parma 2 (1951), 203-213].
\end{abstract}

\section{INTRODUCTION}

In 1981 E. M. Stein proved that if the gradient $\nabla F$ in the distribution sense of a real function $F$ on $\mathbb{R}^{n}$ belongs to the Lorentz space $L(n, 1)$, then $F$ is differentiable at almost all the points in $\mathbb{R}^{n}, n>1$. He further proved that no condition on $\nabla F$ weaker than $\|\nabla F\|_{n, 1}^{*}<\infty$ will guarantee the differentiability of $F$ a.e. in $\mathbb{R}^{n}$, [8]. E. M. Stein refers to "local" $L(n, 1)$; nevertheless the connection between "local" and "global" should be clear to the reader in this context.

Earlier, in 1951, A. P. Calderón had proved that if $\nabla F$ belongs to the Orlicz class

$$
L_{\Psi}=\left\{f: \int_{B} \Psi(|f(x)|) d x<\infty\right\}
$$

with $B$ a ball in $\mathbb{R}^{n}$ and $\Psi$ satisfying

$$
\int_{1}^{\infty}(t / \Psi(t))^{1 /(n-1)} d t<\infty
$$

$\Psi(t)$ nonnegative, nondecreasing, then $F$ is differentiable at almost all the points of $B$. A. P. Calderón further showed that no condition on $\nabla F$ weaker than (1.1), (1.2) above guarantees the a.e. differentiablity on $B$ 1]. Since Calderón's proof suggests that convexity may not be necessary for $\Psi$, we will not require it in what follows. We will refer to those classes of functions as Orlicz type classes.

The aim of this paper is to establish the connection between the Lorentz space $L(p, r), 0<r<p, p>1$, and Orlicz type classes that satisfy a condition akin to 1.2 above. The case $p=n, r=1$ is of particular interest as it implies that the differentiability conditions discussed above are equivalent [2].

2020 Mathematics Subject Classification. $46 \mathrm{E} 30$. 
In a related context, since both Lorentz and Orlicz spaces, as well as the hybrid Lorentz-Orlicz spaces, arise as intermediate spaces of $L^{p}$ spaces [9], it is also of interest to describe their interconnections. Now, if $I=[0,1]$ denotes the unit interval in $\mathbb{R}, L^{p}(I)$ cannot be expressed as the union of the $L^{q}(I)$ spaces it contains properly; $f(x)=|x|^{-1 / p} \ln ^{-2 / p}(1 /|x|) \chi_{I} \in L^{p}(I)$ and $f \notin L^{q}(I)$ for $p<q \leq \infty$. On the other hand, Welland showed that $L^{p}(I)$, as well as more general Orlicz spaces on $I$, can be represented as the union of Orlicz spaces that they contain properly [10]. Since the Lorentz spaces are monotone with respect to the second index [4], and since $L(p, r)(I) \subset L(p, p)(I)=L^{p}(I)$ in the range that is of interest to us, Welland's result gives that $L(p, r)(I)$ can be described as the union of Orlicz spaces that it contains, but this is insufficient to us. Our result covers $L(p, r)\left(\mathbb{R}^{n}\right)$ and, more to the point, the Orlicz type classes $L_{\Psi}$ are taken over the family of functions $\Psi$ that satisfy condition 1.3 below.

We will work with classes of measurable functions $f$ defined on $\mathbb{R}^{n}$. Let $f^{*}$ denote the nonincreasing rearrangement of $|f|$, and let $L(p, r)$, 4, 7], denote the Lorentz space of measurable functions $f$ whose nonincreasing rearrangement $f^{*}$ satisfies

$$
\int_{0}^{\infty} f^{*}(t)^{r} t^{r / p-1} d t<\infty
$$

We will restrict ourselves to the range $0<r<p, p>1$.

Also consider the Orlicz type class $L_{\Psi}$ of measurable functions $f$ defined on $\mathbb{R}^{n}$, such that the rearrangement $f^{*}$ of $|f|$ satisfies

$$
\int_{0}^{\infty} \Psi\left(f^{*}(t)\right) d t<\infty
$$

for a nondecreasing $\Psi \geq 0$ defined on $(0, \infty)$ and satisfying

$$
\int_{0}^{\infty} \frac{t^{q-1}}{\Psi(t)^{q / p}} d t<\infty
$$

where $0<r<p, p>1$, and $1 / p+1 / q=1 / r$.

The aim of this paper is to prove that for $0<r<p, p>1$,

$$
L(p, r)=\bigcup_{\Psi} L_{\Psi}, \quad 1 / p+1 / q=1 / r .
$$

Or, in other words, $f \in L(p, r)$ if and only if

$$
\int_{0}^{\infty} \Psi\left(f^{*}(t)\right) d t<\infty
$$

for some $\Psi$ that satisfies $(1.3)$ above.

The proof is accomplished in two parts, each dealing with an inclusion in (1.4). We only point out that the constants $c$ that appear below may vary from occurrence to occurrence, and are independent of $f$. 


\section{Embedding of Orlicz type Classes into LoREntZ SPACES}

We begin by showing that the Orlicz type classes corresponding to functions $\Psi$ that satisfy 1.3 above are continuously included in an appropriate Lorentz space. More precisely, we have

Proposition 2.1. Let $f$ be a nonnegative, nonincreasing function defined on $(0, \infty)$ such that

$$
\int_{0}^{\infty} \Psi(f(t)) d t<\infty
$$

where $\Psi(t)$ satisfies 1.3 above.

Then, we have

$$
\int_{0}^{\infty} f(t)^{r} t^{r / p-1} d t \leq c\left(\int_{0}^{\infty} \frac{t^{q-1}}{\Psi(t)^{q / p}} d t\right)^{r / q}\left(\int_{0}^{\infty} \Psi(f(t)) d t\right)^{r / p} .
$$

Proof. Let

$$
J=\int_{0}^{\infty} f(t)^{r} t^{r / p-1} d t
$$

and consider the interval $I_{k}$ where $2^{k}<f \leq 2^{k+1},-\infty<k<\infty$. Clearly

$$
J \leq \sum_{k} 2^{(k+1) r} \int_{I_{k}} t^{r / p-1} d t \leq(p / r) \sum_{k} 2^{(k+1) r}\left|I_{k}\right|^{r / p} .
$$

Now, multiplying and dividing by $\Psi\left(2^{k}\right)^{r / p}$, by Hölder's inequality with conjugate indices $(p / r, q / r)$, it readily follows that the sum in the right-hand side of (2.2) is dominated by

$$
\begin{aligned}
\sum_{k} 2^{(k+1) r} \Psi\left(2^{k}\right)^{-r / p}\left|I_{k}\right|^{r / p} \Psi\left(2^{k}\right)^{r / p} & \\
& \leq\left(\sum_{k} \frac{2^{(k+1) q}}{\Psi\left(2^{k}\right)^{q / p}}\right)^{r / q}\left(\sum_{k}\left|I_{k}\right| \Psi\left(2^{k}\right)\right)^{r / p} .
\end{aligned}
$$

Consider the sum in the first factor in 2.3 above. Each summand there can be estimated by

$$
\frac{2^{(k+1) q}}{\Psi\left(2^{k}\right)^{q / p}} \leq c \int_{2^{k-1}}^{2^{k}} \frac{t^{q-1}}{\Psi(t)^{q / p}} d t
$$

and, consequently, the sum does not exceed

$$
c \sum_{k} \int_{2^{k-1}}^{2^{k}} \frac{t^{q-1}}{\Psi(t)^{q / p}} d t=c \int_{0}^{\infty} \frac{t^{q-1}}{\Psi(t)^{q / p}} d t .
$$

As for the second sum, since

$$
\left|I_{k}\right| \Psi\left(2^{k}\right) \leq \int_{I_{k}} \Psi(f(t)) d t
$$

it readily follows that

$$
\sum_{k}\left|I_{k}\right| \Psi\left(2^{k}\right) \leq \int_{0}^{\infty} \Psi(f(t)) d t .
$$


Thus, combining (2.2), 2.3), (2.4), and (2.5) above, the estimate (2.1) holds, and the proof is finished.

\section{EMbedding of LoRentz SPACES INTO ORLiCZ TYPe ClASSES}

We complete the proof by showing that if $f \in L(p, r)$ for an appropriate range of values of $p, r$, then $f$ is in an Orlicz type class $L_{\Psi}$, where $\Psi$ depends on $f$. More precisely, we have

Proposition 3.1. Let $0<r<p, p>1$. Let $f$ be a nonnegative, nonincreasing function defined on $(0, \infty)$ such that

$$
\int_{0}^{\infty} f(t)^{r} t^{r / p-1} d t<\infty
$$

Then, with $1 / p+1 / q=1 / r$, there exists a nonnegative, nondecreasing function $\Psi(t)$ defined on $(0, \infty)$ satisfying $(1.3)$ above for which

$$
\int_{0}^{\infty} \Psi(f(t)) d t<\infty
$$

Proof. Let $f_{0}(t)$ be a strictly positive, strictly decreasing function on $(0, \infty)$ such that

$$
\lim _{t \rightarrow 0^{+}} f_{0}(t)=\infty, \quad \lim _{t \rightarrow \infty} f_{0}(t)=0,
$$

and

Let now $g_{0}=f+f_{0}$. Then,

$$
\int_{0}^{\infty} f_{0}(t)^{r} t^{r / p-1} d t<\int_{0}^{\infty} f(t)^{r} t^{r / p-1} d t
$$

$$
f(t)<g_{0}(t), \quad \text { for all } t
$$

and

$$
\int_{0}^{\infty} g_{0}(t)^{r} t^{r / p-1} d t \leq \max \left(2,2^{r}\right) \int_{0}^{\infty} f(t)^{r} t^{r / p-1} d t<\infty .
$$

Finally, we define the function $g(t)$. Let $J_{k}$ be the interval where $2^{k}<g_{0}(t) \leq$ $2^{k+1}$, and $\left[a_{k}, b_{k}\right]$ its closure. Then $g(t)$ is defined by

$$
g\left(a_{k}\right)=2^{k+1}, \quad g\left(b_{k}\right)=2^{k}
$$

and extended linearly on $\left[a_{k}, b_{k}\right]$. It follows that $g(t)$ is strictly decreasing, continuous on $(0, \infty)$, absolutely continuous, invertible, and

$$
g_{0}(t) / 2<g(t)<2 g_{0}(t) .
$$

Furthermore, since $g(t)$ is decreasing and $r<p$ it follows that

$$
g(\varepsilon)^{r} \varepsilon^{r / p} \leq \int_{0}^{\varepsilon} g(t)^{r} t^{r / p-1} d t, \quad \varepsilon>0
$$

and, consequently,

$$
\lim _{\varepsilon \rightarrow 0^{+}} g(\varepsilon)^{r} \varepsilon^{r / p}=0 .
$$


Likewise, for large $N$, we have

$$
g(N)^{r} N^{r / p} \leq c \int_{N / 2}^{N} g(t)^{r} t^{r / p-1} d t,
$$

and, consequently, since $\int_{0}^{\infty} g(t)^{r} t^{r / p-1} d t<\infty$,

$$
\lim _{N \rightarrow \infty} g(N)^{r} N^{r / p}=0 .
$$

Let now $\Psi(t)$ be defined by the equation

$$
\Psi(g(t))=g(t)^{r} t^{r / p-1},
$$

and let $\varphi(t)$ be given by

$$
\Psi(t)=t^{r} \varphi(t)
$$

From 3.7 and 3.8 it follows that

$$
\varphi(g(t))=t^{r / p-1} .
$$

This gives that $\varphi(t)$ increases, and, consequently, $\Psi(t)$, and $\Psi(t) / t^{r}$, are increasing.

Next we verify that $\Psi$ satisfies 1.3 . Since $\Psi(t)=t^{r} \varphi(t)$ and $r / p+r / q=1$, by (3.9) it follows that

$$
\int_{0}^{\infty} \frac{t^{q-1}}{\Psi(t)^{q / p}} d t=\int_{0}^{\infty} \frac{t^{q-1} t^{r-q}}{\varphi(t)^{q / p}} d t=\int_{0}^{\infty} t^{r-1} \varphi(t)^{1-q / r} d t .
$$

By the substitution $t=g(u)$, since $r / p-1=-r / q$ and $1-q / r=-q / p$, the right-hand side of $(3.10)$ becomes

$$
-\int_{0}^{\infty} g(u)^{r-1}\left(u^{r / p-1}\right)^{1-q / r} g^{\prime}(u) d u=-\int_{0}^{\infty} g(u)^{r-1} g^{\prime}(u) u^{r / p} d u .
$$

Now, on account of 3.5 and (3.6), integration by parts gives that 3.11 evaluates to

$$
c \int_{0}^{\infty} g(u)^{r} u^{r / p-1} d u
$$

which by $(3.3)$ and $(3.4)$ is finite, and $(1.3)$ holds.

Moreover, by (3.7) it follows that

$$
\int_{0}^{\infty} \Psi(g(u)) d u<\infty
$$

and, consequently, by 2.5 and 3.2 ,

$$
\int_{0}^{\infty} \Psi(f(u) / 2) d u \leq \int_{0}^{\infty} \Psi\left(g_{0}(u) / 2\right) d u \leq \int_{0}^{\infty} \Psi(g(u)) d u<\infty .
$$

Repeating the above argument with $2 f$ replacing $f$ above, 3.12 becomes

$$
\int_{0}^{\infty} \Psi(2 f(u) / 2) d u=\int_{0}^{\infty} \Psi(f(u)) d u<\infty,
$$

(3.1) holds, and the proof is finished. 
Since as noted in the proof $\Psi(t) / t$ increases when $r=1, \Psi(t)$ can be regularized to a convex function $\Psi_{0}(t)$ such that $\Psi_{0}(t) \leq \Psi(t) \leq \Psi_{0}(2 t)$, and, therefore, in this case the Orlicz type class $L_{\Psi}$ is essentially equivalent to an Orlicz space [5, 9].

\section{REFERENCES}

[1] A. P. Calderón, On the differentiability of absolutely continuous functions, Riv. Mat. Univ. Parma 2 (1951), 203-213. MR 0045200

[2] C. P. Calderón, A characterization of the Lorentz space $L(p, 1)$ in terms of Orlicz type classes, preprint, 2019.

[3] C. P. Calderón and J. Lewis, Maximal smoothing operators and some Orlicz classes, Studia Math. 57 (1976), no. 3, 285-296. MR 0448060

[4] R. A. Hunt, On $L(p, q)$ spaces, Enseign. Math. (2) 12 (1966), 249-276. MR 0223874

[5] M. Jodeit, Jr., Some relations among Orlicz spaces, M.A. Thesis, Rice University, 1965. https://hdl.handle.net/1911/89749.

[6] M. Nowak, Inductive limit topologies on Orlicz spaces, Comment. Math. Univ. Carolin. 32 (1991), no. 4, 667-675. MR 1159813

[7] N. M. Rivière, Interpolation à la Marcinkiewicz, Rev. Un. Mat. Argentina 25 (1970/71), 363-377. MR 0370169

[8] E. M. Stein, Editor's note: the differentiability of functions in $\mathbf{R}^{n}$, Ann. of Math. (2) 113 (1981), no. 2, 383-385. MR 0607898

[9] A. Torchinsky, Interpolation of operations and Orlicz classes, Studia Math. 59 (1976/77), no. 2, 177-207. MR 0438105

[10] R. Welland, Inclusion relations among Orlicz spaces, Proc. Amer. Math. Soc. 17 (1966), 135-139. MR 0188773

C. P. Calderón

Dept of Math, Stat \& Comp Sci, University of Illinois at Chicago, Chicago IL 60607, USA

cpc@uic.edu

A. Torchinsky ${ }^{凶}$

Dept of Math, Indiana University, Bloomington IN 47405, USA

torchins@indiana.edu

Received: December 11, 2019

Accepted: February 12, 2020 